\title{
Resonance Analysis of High-Frequency Electrohydraulic Exciter Controlled by 2D Valve
}

\author{
Guojun Pan, ${ }^{1}$ Yan Ren, ${ }^{2}$ and Jian Ruan $^{3}$ \\ ${ }^{1}$ College of Information and Engineering, Zhejiang Radio \& Television University, Hangzhou 310030, China \\ ${ }^{2}$ College of Mechanical \& Electrical Engineering, Wenzhou University, Wenzhou 325035, China \\ ${ }^{3}$ Key Laboratory of Special Purpose Equipment and Advanced Machining Technology, Ministry of Education \& Zhejiang Province, \\ Zhejiang University of Technology, Hangzhou 310014, China
}

Correspondence should be addressed to Yan Ren; rentingting211@163.com

Received 18 November 2014; Accepted 18 December 2014

Academic Editor: Yanxue Wang

Copyright (c) 2015 Guojun Pan et al. This is an open access article distributed under the Creative Commons Attribution License, which permits unrestricted use, distribution, and reproduction in any medium, provided the original work is properly cited.

The resonant characteristic of hydraulic system has not been described yet because it is necessarily restricted by linear assumptions in classical fluid theory. A way of the resonance analysis is presented for an electrohydraulic exciter controlled by $2 \mathrm{D}$ valve. The block diagram of this excitation system is established by extracting nonlinear parts from the traditional linearization analysis; as a result the resonant frequency is obtained. According to input energy from oil source which is equal to the reverse energy to oil source, load pressure and load flow are solved analytically as the working frequency reaches the natural frequency. The analytical expression of resonant peak is also derived without damping. Finally, the experimental system is built to verify the theoretical analysis. The initial research on resonant characteristic will lay theoretical foundation and make useful complement for resonance phenomena of classical fluid theory in hydraulic system.

\section{Introduction}

Material fatigue or crack growth is a phenomenon commonly met in mechanical engineering practice $[1,2]$. Thus, fatigue testing becomes an important and wise means to objectively determine the performance of a mechanical product working under vibrating condition [3]. Recently high-cycle fatigue testing is more and more of interest. In Wright Patterson Air Force Base, an electrodynamic shaker has been designed to study high-frequency characteristics, of which working frequency is from $350 \mathrm{~Hz}$ to $600 \mathrm{~Hz}$ depending on the system itself. And a magnetostrictive material as an actuator is used in electromagnetic exciter in order that working frequency is up to about $2 \mathrm{kHz}$ [4]. In Southwest Research Institute, a piezoelectric driver is used in a new exciter for enhancing frequency bandwidth in a large scale. Working frequency, as a function of the specimen and grips, can be achieved near $2 \mathrm{kHz}$, but it is limited to the resonance point [5]. The vibrating enviroment is created artificially by an exciter, which is driven by mechanical power, electrical power, or hydraulic power [6]. However, in the case of heavy power, an electrohydraulic exciter is commonly used. An electrohydraulic exciter is designed by MTS specifically for high-cycle fatigue testing [7]. It is controlled by a two-stage servo valve. Though working frequency is improved greatly, flow capacity is limited because of the structure of nozzle flapper. So a voice valve with higher flow rate and frequency response is used in an electrohydraulic exciter designed by MTS Corporation. This system is integrated for elastomeric material testing and crack growth testing in Michigan Technological University. This exciter's operating frequency is increased (1000 Hz or above), but voice coil motor requires additional cooling device to reduce the temperature under a high current working environment. A three-stage servo valve is produced by Rexroth [8]. It has better dynamic response than nozzle-flapper structure, but the bandwith of big flow valve is about $200 \mathrm{~Hz}$. In addition to designing new electromechanical transformer and two-stage or three-stage structure of servo valve, new control components have been tried to replace servo valve used in electrohydraulic exciter. 
Hao and Zhang [9] used a rotary valve in exciter to improve vibration frequency. By controlling this rotary valve, the spool will make a reciprocating motion to switch pressurized flow rate to chambers of the cylinder or motor alternatively and thus a vibration is created to the piston of the cylinder and connected load. But vibration amplitude was dominated by the oil's pressure and thus had low accuracy, and the offset control was difficult to achieve.

The hydraulic exciter is much restrained by the dynamic performance of the servo valve, which is difficult to enhance working frequency to a very high level. A novel scheme for an electrohydraulic exciter controlled by a $2 \mathrm{D}$ valve is therefore proposed to achieve higher frequency. The frequency and the amplitude of sinusoidal vibration wave are separately controlled by circumferential rotation and axial sliding of spool, respectively [10]. But the relationship of vibration frequency and the amplitudes is intercoupling; real data is scarce. Therfore a promising solution is increasing working frequency up to natural frequency of hydraulic system. When frequency of the excited vibration comes close to the natural frequency, resonance occurs and can improve vibration amplitude greatly. This hydraulic resonance will be researched and discussed through theoretical analysis and experimental system, and finally some conclusions are given.

\section{Working Principle}

$2 \mathrm{D}$ valve, as a necessary control component, is used in high-frequency electrohydraulic exciter because of this valve's special structure. $2 \mathrm{D}$ valve has two degrees of freedom, the rotational and sliding motions of the spool. Such structure not only acts a two-stage valve with pilot stage and direct position feedback but also greatly improves the excited frequency by increasing spool's rotational speed. The spool is continuously rotated by an electric motor and gear combination and sliding by an eccentric mechanism driven by other electric motors, as shown in Figure 1.

The rotation of the $2 \mathrm{D}$ valve spool can produce the alternatively varied valve port area which is formed between the grooves on a spool land and the rectangular windows on the sleeve. There is a series of grooves symmetrically distributed on the spools (central angle of every groove is $\alpha$ ) coordinated with the windows distributed uniformly on the sleeve which create an "alternately varied" valve port area, as shown in Figure 2. Then geometrically, the groove has $2 \alpha$ angle difference with respect to one on any two adjacent shoulders. Such arrangement will cause a waveform with $180^{\circ}$ phase angle between the meter-in valve port and the meter-out valve port. The varied valve port area depends on rotary speed and axial sliding of the spool. Consequently the electrohydraulic vibrator controlled by $2 \mathrm{D}$ valve could realize that the frequency and the amplitude of vibration are dominated, respectively, by the rotary speed and the sliding displacement of the spool of $2 \mathrm{D}$ valve.

The combination of $2 \mathrm{D}$ valve and a piston is selected as a hydraulic power element to produce a reciprocating motion. One chamber of the biactuation cylinder is controlled by

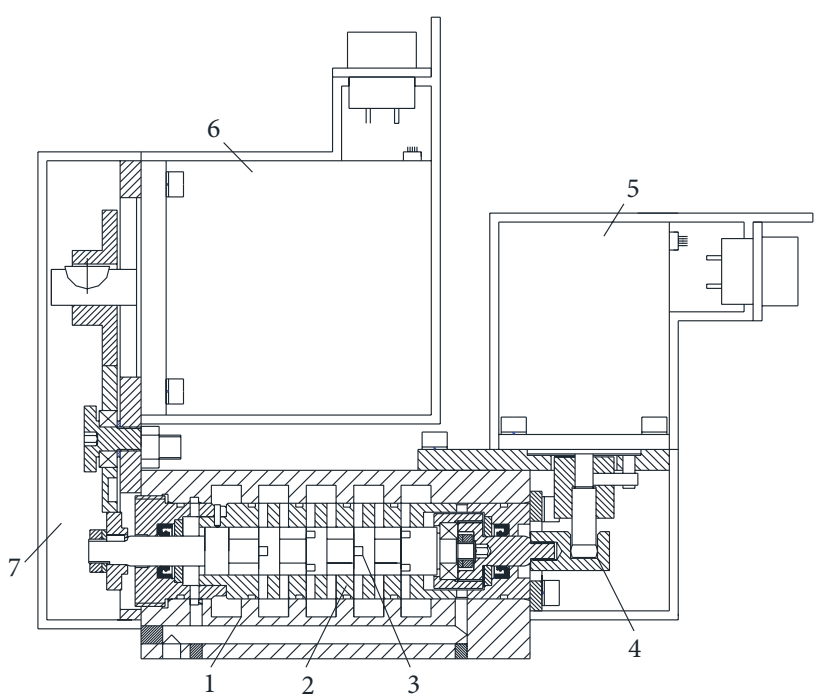

FIGURE 1: 2D valve with two motors. 1: valve body, 2: sleeve, 3: spool, 4: eccentric mechanism, 5: stepping motor, 6: electric motor, and 7: gearbox.

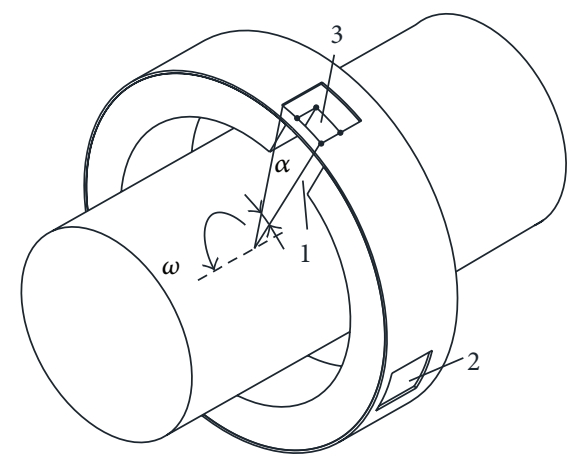

FIGURE 2: Coupling pattern of 2D valve. 1: groove on the spool, 2: window on the sleeve, and 3: rectangular area.

a hydraulic bridge formed by meter-in and meter-out alternatively varied valve ports. Another chamber is a hydraulic bridge with a $180^{\circ}$ phase angle difference. As the spool of the $2 \mathrm{D}$ valve makes a rotary motion the pressure inside two chambers of the cylinder will change alternatively to make the piston output a reciprocating vibration by reference to Figures 3(a) and 3(b). Obviously, it is easy to obtain highfrequency vibration by improving rotary speed of the spool. The working frequency is also related to the number of the grooves on a single spool land and the coupling pattern between the grooves on the spool land and the windows on the sleeve. However, this is achieved at the price of sacrificing the magnitude of the $2 \mathrm{D}$ valve orifice area that decides the amplitude of vibration. So in high-frequency section, the excited amplitude will be to a large extent limited. 


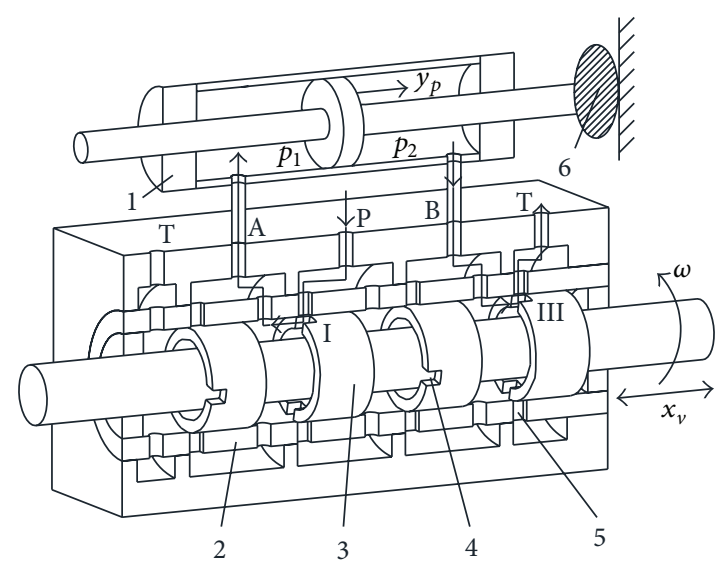

(a)

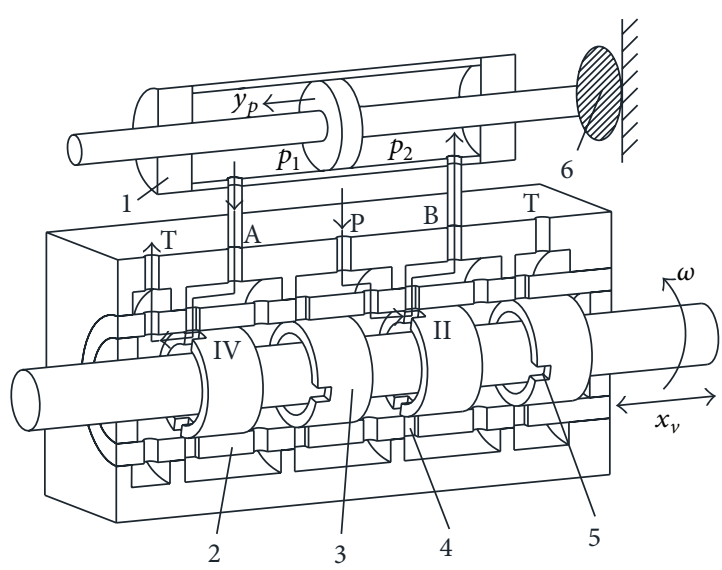

(b)

FIGURE 3: Valve ports I and III open; piston moves to the right (a), and valve ports II and IV open; piston moves to the left (b). 1: cylinder, 2: sleeve, 3: spool, 4: window, 5: groove, and 6: excited object.

\section{Resonant Characteristics}

The resonant principle of hydraulic fluid and mechanical system is thoroughly different because the elements of mechanical resonance including mass, damping, and spring are mutually independent and the relevance among them is very weak. However, hydraulic resonance elements composed of spring, damping, and flow force are almost made with flow power transmission mediums except mass. This hydraulic medium that is working fluid not only acts as a force to drive cycle oscillation of the piston, but also is a centering spring of stiffness is a function of piston displacement and takes into effect like a viscous damping because of a leakage path acting to increase damping. Therefore this hydraulic resonance will come out with peculiar resonant phenomenon and theoretical results.

3.1. Resonance Process. This exciter producing hydraulic resonance is under a condition that working frequency should reach system natural frequency. So acceleration of the piston will be greatly increased so that the piston still moves in the left-hand direction even though passing by the center position of hydraulic cylinder when valve ports 1 and 3 are open (the orifice areas are denoted by $A_{v 1}$ and $A_{v 3}$ ), where oil is directed to the left cylinder chamber and then drained from the right cylinder chamber to the tank (the flow rates are, resp., $Q_{\nu 1}$ and $Q_{\nu 3}$ ). This leads to the fact that the volume of the left chamber tapers off and then the pressure of the left chamber $p_{1}$ gradually increases. As a result, the oil in this chamber is compressed until the pressure of the left chamber $p_{1}$ is higher than the system pressure $p_{s}$, as shown in Figure $4(\mathrm{a})$. When $p_{1}$ is high enough, a phenomenon about oil backward will appear, because this pressure exceeding the system pressure is so high that the oil retrogrades back into the tank, as shown in Figure 4(b). Then the compressed oil starts to expand gradually so that the inertia force is reduced to zero and then the piston is driven to make reverse motion, which could be obtained from arrowhead direction of displacement $y_{p}$ in Figure 4(c). This makes the volume of left chamber be higher and the pressure of left chamber decrease. Until $p_{1}$ becomes lower than $p_{s}$, the oil stops backing into the tank and repeats to enter the left cylinder chamber and exit from the right cylinder to drive the piston moving to the right, as shown in Figure 4(d). When the piston moves to the center position of hydraulic cylinder, valve ports 1 and 3 are closed.

As the spool of $2 \mathrm{D}$ valve spins, the high-pressure oil flows into the right chamber of the cylinder through the valve port 2 and out from the left chamber through the valve port 4 (valve ports 2 and 4 are open; the orifice areas are denoted by $A_{v 2}$ and $A_{v 4}$ ); the piston is driven to move rightward under the effect of inertia force. The volume of the right chamber decreases and then the pressure of the right chamber $p_{2}$ gradually increases. When this pressure $p_{2}$ is higher than the system pressure $p_{s}$, the phenomenon about oil backward will appear again, as shown in Figures 5(a) and 5(b). Then the compressed oil in the right chamber starts to expand gradually so that inertia force is reduced to zero and then the piston is provided direction reversal. This makes the volume of right chamber become higher and the pressure of right chamber decrease. When $p_{2}$ is lower than $p_{s}$, the oil stops backing into the tank and flows into the right chamber and out of the left chamber (the flow rates are, resp., $Q_{v 2}$ and $\left.Q_{v 4}\right)$ to drive the piston moving to the left. It is illustrated by Figures 5(c) and 5(d). When the piston moves to the center position of hydraulic cylinder, valve ports 2 and 4 are closed and valve ports 1 and 3 are open simultaneously. Consequently, the piston of hydraulic cylinder is driven to make reciprocating motion. A vibration excited is achieved under the condition of resonant frequency.

3.2. Dynamic Analysis. In making a dynamic analysis, it is necessary that the nonlinear algebraic equations which 


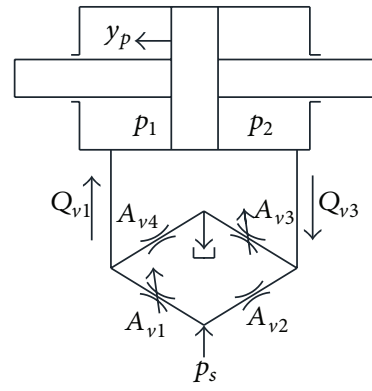

(a)

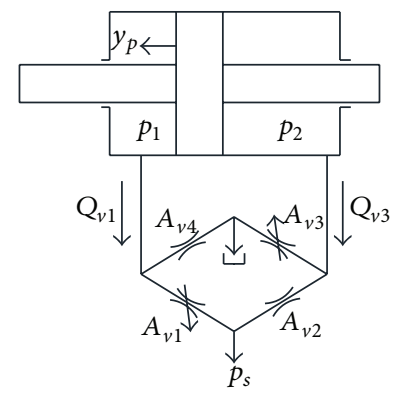

(b)

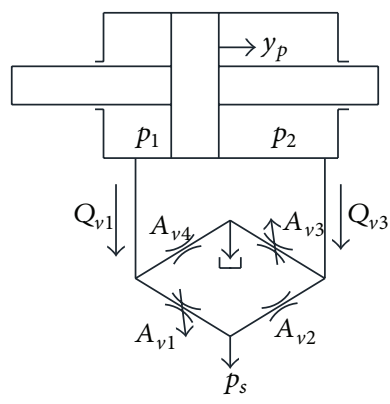

(c)

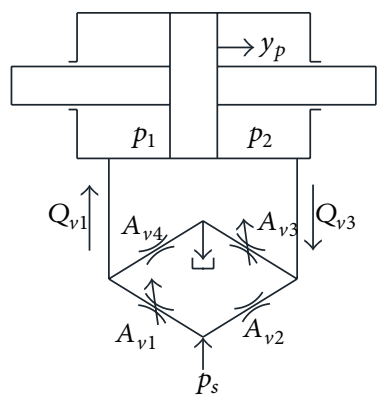

(d)

Figure 4: Resonance process of piston being in the left chamber (a), (b), (c), and (d).

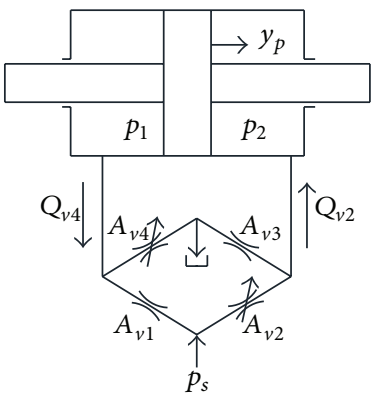

(a)

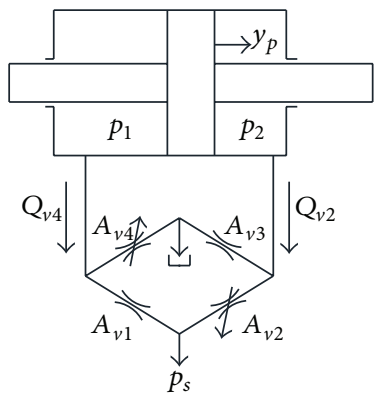

(b)

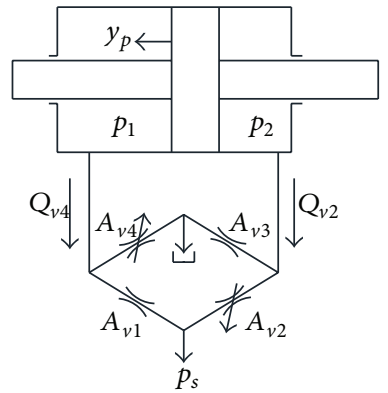

(c)

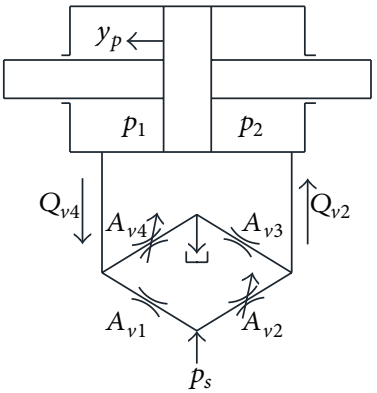

(d)

Figure 5: Resonance process of piston being in the right chamber (a), (b), (c), and (d).

describe the pressure-flow curves be linearized. A general expression for the load flow is

$$
Q_{L}=K_{q} X_{v}-K_{c} P_{L}
$$

where $Q_{L}$ is then flow through the load, $K_{q}$ is the flow gain, $X_{v}$ is the valve position, $K_{c}$ is the flow-pressure coefficient, and $P_{L}$ is the pressure drop across the load.

In the combination of $2 \mathrm{D}$ valve and piston, ideal geometry of $2 \mathrm{D}$ valve ports is completely analogous to matched and symmetrical orifices of critical center servo valve. So applying the continuity equation to each of the piston chambers yields

$$
\begin{aligned}
Q_{L}= & \frac{Q_{1}+Q_{2}}{2} \\
= & k_{i c}\left(p_{1}-p_{2}\right)+\frac{1}{2} k_{e c}\left(p_{1}-p_{2}\right)+\frac{A_{p} d y_{p}}{d t}+\frac{V_{01} d p_{1}}{2 E_{h} d t} \\
& -\frac{V_{02} d p_{2}}{2 E_{h} d t}+\frac{A_{p} y_{p}}{2}\left(\frac{d p_{1}}{d t}+\frac{d p_{2}}{d t}\right),
\end{aligned}
$$

where $Q_{1}, Q_{2}$ are forward and return flows, $p_{1}, p_{2}$ are forward and return pressures, $k_{i c}$ is internal or cross-port leakage coefficient of piston, $k_{e c}$ is external leakage coefficient of piston, $y_{p}$ is displacement of piston, $A_{p}$ is the area of piston, $V_{01}$ is initial volume of forward chamber, $V_{02}$ is initial volume of return chamber, and $E_{h}$ is the bulk modulus of oil.
It is assumed that the piston is centered and the volumes of the piston chambers are equal; that is, $V_{01}=V_{02}=V_{t} / 2$. This assumption is made that vibration amplitude is smaller in high working frequency so that $A_{p} \cdot y_{p}$ is ignored here. Therefore

$$
Q_{L}=\frac{Q_{1}+Q_{2}}{2}=k_{t c} p_{L}+\frac{A_{p} d y_{p}}{d t}+\frac{V_{t} d p_{L}}{4 E_{h} d t},
$$

where $k_{t c}$ is the total leakage coefficient of piston and $V_{t}$ is the total volume of fluid under compression in both chambers.

The volume and continuity expressions can be Laplacetransformed to yield

$$
Q_{L}=A_{p} s Y_{p}+\left[k_{t c}+\frac{V_{t}}{4 E_{h}} s\right] p_{L} .
$$

The resulting force equation, Laplace-transformed, is

$$
p_{L}=\frac{1}{A_{p}}\left[\left(m s^{2}+B_{p} s+K_{L}\right) Y_{p}+F_{L}\right],
$$

where $m$ is the total mass of piston and load referred to piston, $B_{p}$ is viscous damping coefficient of piston and load, $K_{L}$ is load spring gradient, and $F_{L}$ is arbitrary load force on piston.

The three basic equations (1), (4), and (5) are represented in block diagram fashion in Figure 6. 


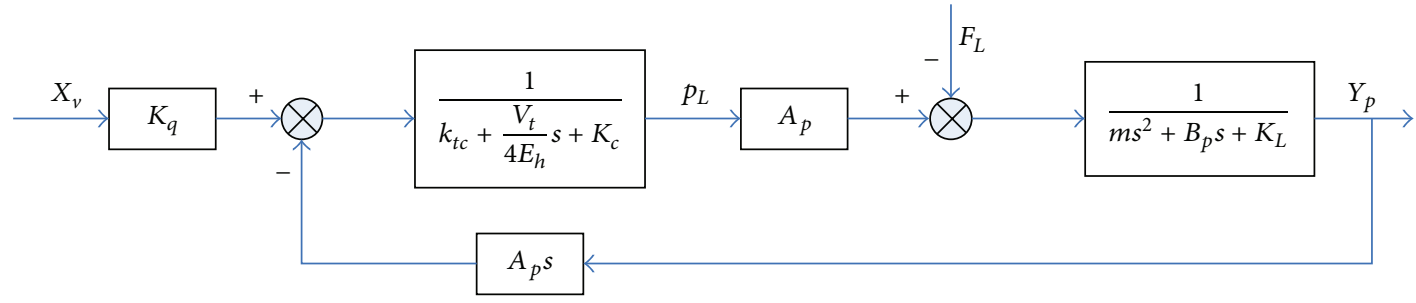

FIGURE 6: Diagram based on obtaining piston position from flow.

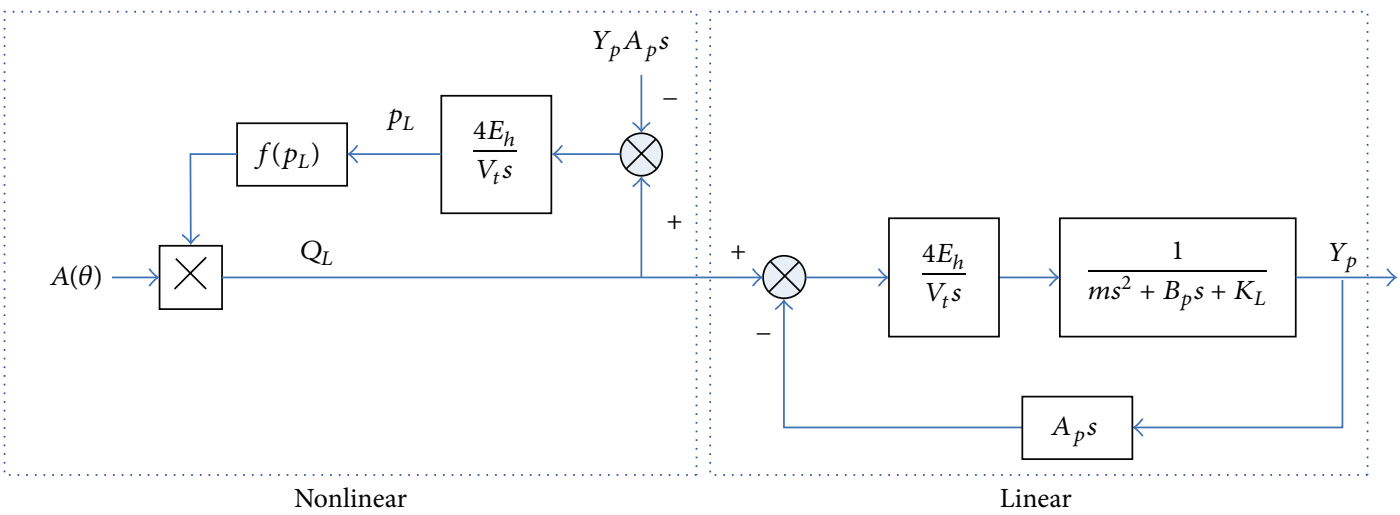

FIGURE 7: Block diagram of electrohydraulic exciter controlled by 2D valve.

The load flow as a function of valve position and load pressure being linearized can give a complete description of steady-state valve performance. However, in electrohydraulic excited system controlled by 2D, the load pressure with highfrequency period variations has not been satisfied for linear conditions. So the block diagram of this model is shown in Figure 7.

The transfer function for this condition is

$$
\begin{aligned}
\frac{Y_{p}}{Q_{L}}= & \left(\frac{4 E_{h} A_{p}}{\left(V_{t} K_{L}+4 E_{h} A_{p}^{2}\right)}\right) \\
& \times\left(s \left(\frac{s^{2}}{\left(V_{t} K_{L}+4 E_{h} A_{p}^{2}\right) /\left(V_{t} m\right)}\right.\right. \\
& \left.\left.\quad+\frac{s}{\left(V_{t} K_{L}+4 E_{h} A_{p}^{2}\right) /\left(V_{t} B_{p}\right)}+1\right)\right)^{-1} \\
= & \frac{K_{V}}{s\left(s^{2} / \omega_{0}^{2}+\left(2 \xi_{0} s\right) /\left(\omega_{0}\right)+1\right)} .
\end{aligned}
$$

Analysis of describing function equation (6) yields the resonant frequency:

$$
\omega_{0}=\sqrt{\frac{V_{t} K_{L}+4 A_{p}^{2} E_{h}}{V_{t} m} .}
$$

Obviously, the resonant frequency for electrohydraulic exciter controlled by $2 \mathrm{D}$ valve consists of hydraulic natural part and mechanical part.

3.3. Resonant Peak. There is resonance in this electrohydraulic exciter system when working frequency is increased to system natural frequency. This phenomenon causes excited output oscillating with a fixed amplitude and frequency. Because excited waveform just contains a dominant frequency but no other harmonics, it is assumed as follows:

$$
y_{p}=-A_{p f} \sin (z \theta),
$$

where $A_{p f}$ is resonant peak.

And the pressure drop across the load under resonant frequency is

$$
p_{L}=p_{L f} \sin (z \theta)
$$

where $p_{L f}$ is amplitude of load pressure.

As discussed in Section 3.1, flow equations are rewritten as

$$
Q_{L}= \begin{cases}\operatorname{sign}\left(p_{s}-p_{L}\right) C_{0} A_{v 1} \sqrt{\left|p_{s}-p_{L}\right|} & \theta \in[0,2 \alpha] \\ -\operatorname{sign}\left(p_{s}+p_{L}\right) C_{0} A_{v 2} \sqrt{\left|p_{s}+p_{L}\right|} & \theta \in[2 \alpha, 4 \alpha],\end{cases}
$$




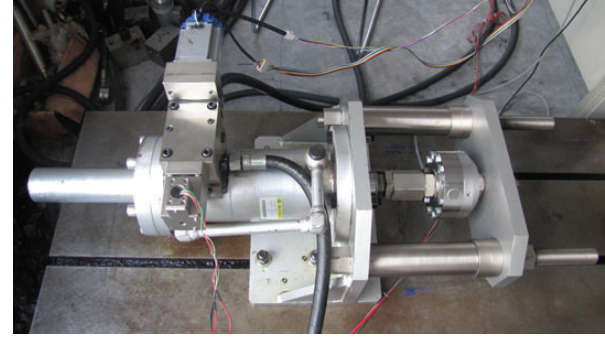

(a)

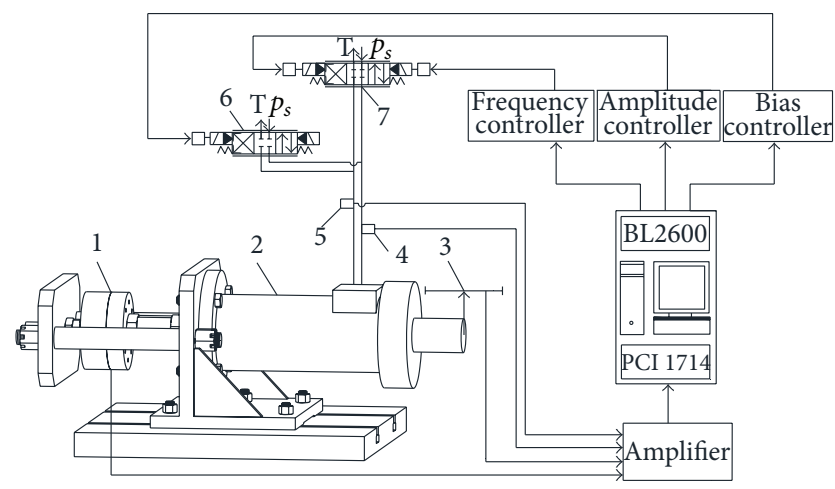

(b)

FIGURE 8: Photo of experimental system (a) and schematic diagram of experimental system of high-frequency electrohydraulic exciter (b). 1: force cell, 2: cylinder, 3: displacement transducer, 4: pressure sensor for the left chamber, 5: pressure sensor for the right chamber, 6: servo valve, and $7: 2 \mathrm{D}$ valve.

where $C_{0}$ is a constant related to flow rate coefficient and oil density.

On the basis of energy conservation input energy is equal to output energy in a cycle; that is,

$$
\sum_{1}^{4} I_{i}=\int_{0}^{4 \alpha} Q_{L} p_{L} d \theta=0
$$

where

$$
\begin{aligned}
& I_{1}+I_{2} \\
& =\int_{0}^{2 \alpha} p_{L f} \sin (z \theta) \operatorname{sign}\left(p_{s}-p_{L f} \sin (z \theta)\right) \\
& \quad \times C_{0} A_{v 1} \sqrt{\left|p_{s}-p_{L f} \sin (z \theta)\right|} d \theta, \\
& I_{3}+I_{4} \quad \int_{2 \alpha}^{4 \alpha}-p_{L f} \sin (z \theta) \operatorname{sign}\left(p_{s}+p_{L f} \sin (z \theta)\right) \\
& \quad \times C_{0} A_{v 2} \sqrt{\left|p_{s}+p_{L f} \sin (z \theta)\right|} d \theta .
\end{aligned}
$$

Equations (9), (10), and (11) can be solved simultaneously to obtain

$$
p_{L f}=w p_{s}
$$

where $w$ is a pressure ratio.

Assuming mass force and spring force dominant, force equation on the piston is

$$
A_{p} p_{L}=m \omega_{0}^{2} \frac{d^{2} y_{p}}{d \theta^{2}}+K_{L} y_{p} .
$$

Therefore these equations may then be combined to yield the resulting resonant peak:

$$
A_{p f}=\frac{V_{t} p_{L f}}{4 A_{p} E_{h}} .
$$

It is clear that resonant peak depends on electrohydraulic exciter system and is irrelevant to input parameters including the orifice areas or shapes of valve ports.

\section{Experiments and Results}

The experimental system is illustrated by reference to Figure 8, which mainly consists of the combination of $2 \mathrm{D}$ valve and piston, acquisition elements and a control part. This exciter is mostly applied to the high-cycle fatigue testing machine, so the load force can be considered as the frame in an axial direction of the cylinder. A displacement transducer is chosen to be mounted inside the cylinder rod to measure the displacement of the piston. A force cell is placed between the end of the cylinder rod and the rigid frame to measure the output force of the cylinder's piston. And two pressure sensors, respectively, are used to acquire the pressures in the right and left chambers. These signals are amplified and then are sent to industrial computer to display, save, and further treat. The frequency and amplitude signals are sent separately to two motors to control the rotary and linear motion of $2 \mathrm{D}$ valve's spool. The bias signal is sent to a servo valve connected in parallel with $2 \mathrm{D}$ valve to control the bias displacement of output vibration.

The partial experimental excited waveforms are shown in Figure 9. And the relationship between vibration amplitude and working frequency is summarized and also presented in this figure. The vibration amplitude descends rapidly with the working frequency increasing but tends to be flat as this frequency is much close to the natural frequency. 


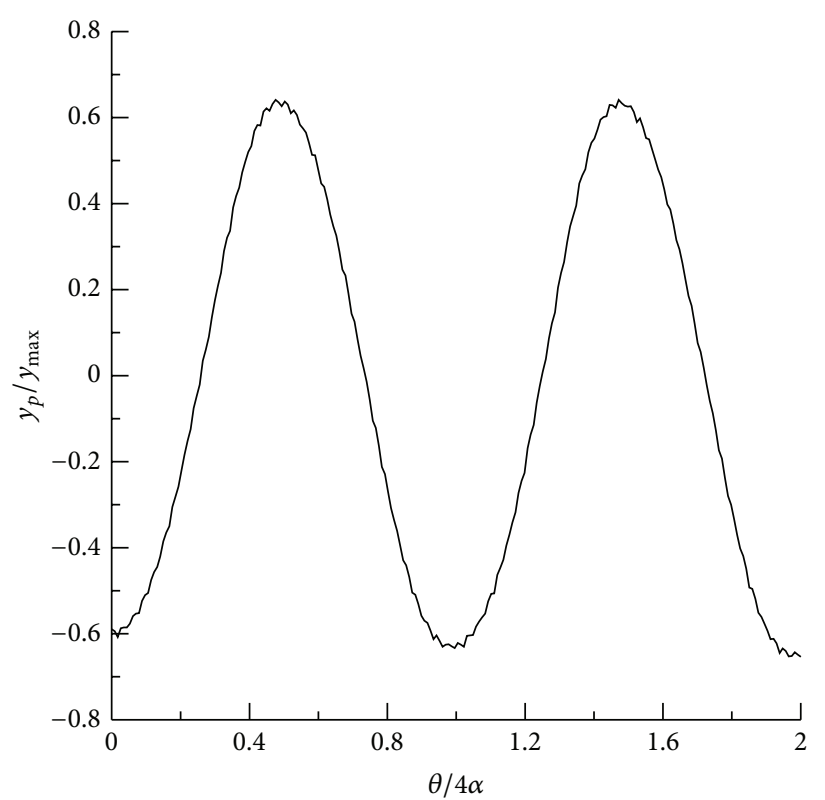

(a)

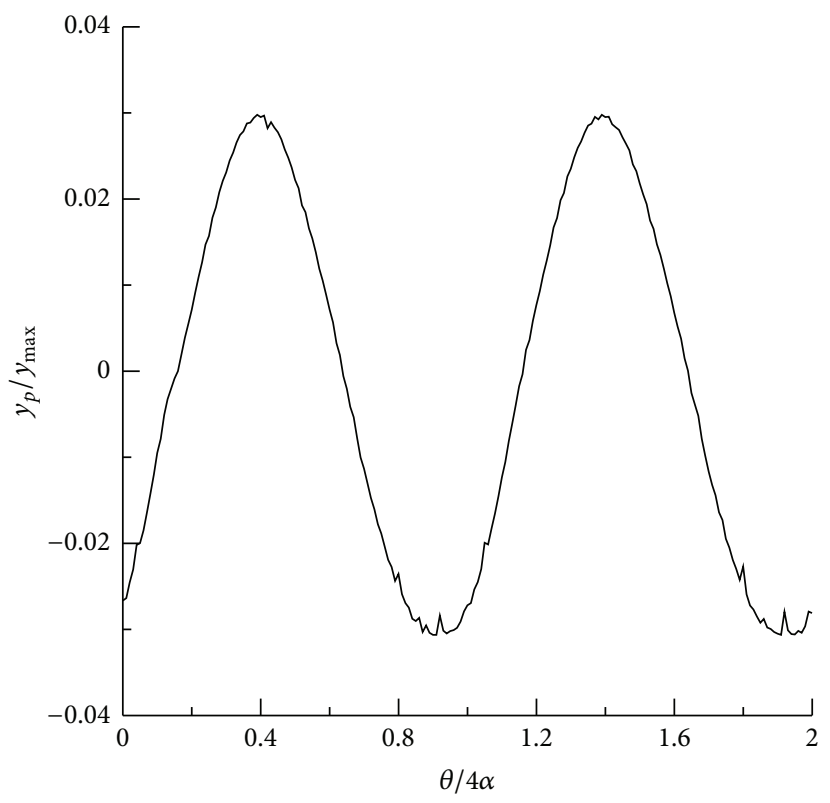

(c)

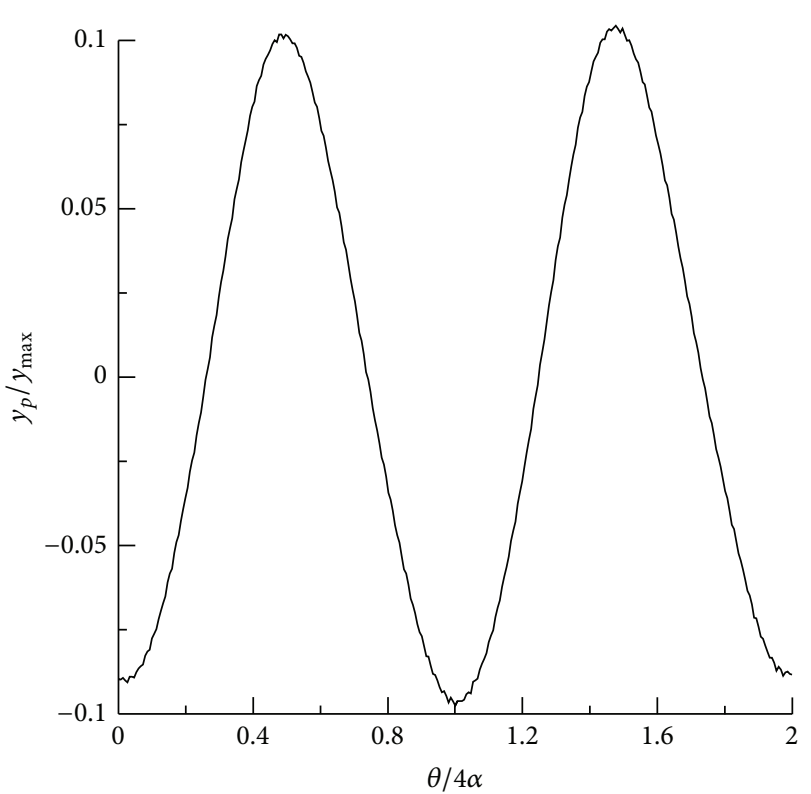

(b)

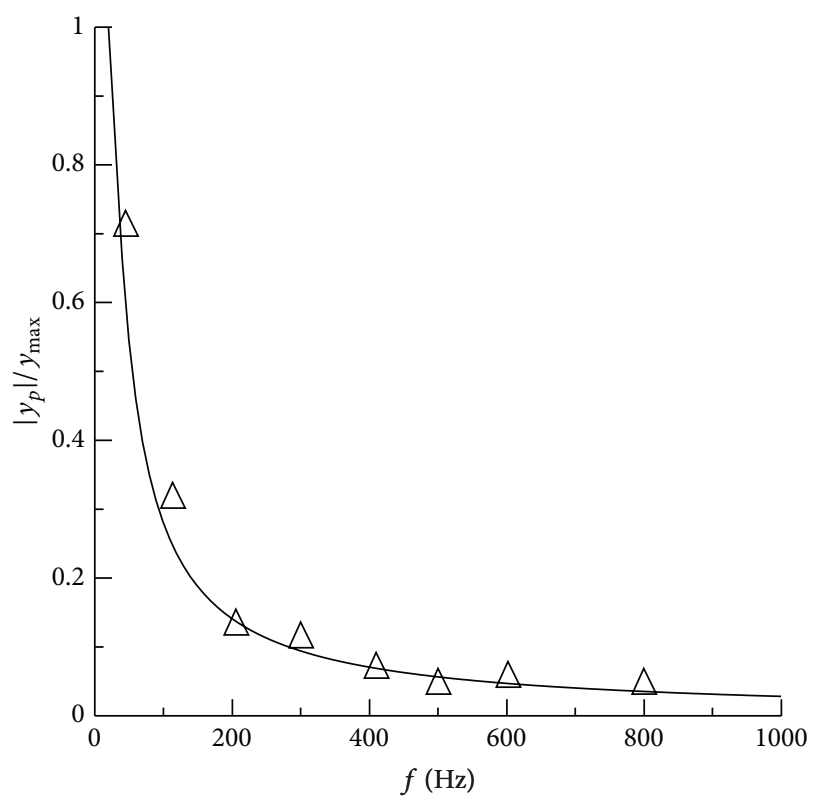

(d)

FIGURE 9: Excited waveform $f=40 \mathrm{~Hz}$ (a), excited waveform $f=300 \mathrm{~Hz}$ (b), excited waveform $f=800 \mathrm{~Hz}$ (c), and amplitude-frequency curve (d).

Primary resonant results are given in Figure 10, which includes the displacement waveform, pressure waveform in each chamber, and frequency composition.

By referring to Figure 10, it can be seen that vibration amplitude is increased obviously at resonant frequency point. But pressure in each chamber is not higher than system pressure, which causes experimental resonant amplitude which is smaller than the theoretical result. A major source is that viscous force is completely ignored in resonant analysis even though it is not dominant and is a soft quantity that is difficult to be measured and computed.

\section{Conclusions}

A 2D valve is adopted to control electrohydraulic exciter for working frequency greatly increasing and even reaching the resonant frequency. Hydraulic resonance is a peculiar resonant phenomenon, so it is necessary to analyse its characteristic especially within the context of energy conservation. Theoretical resonant peak is a constant and its analytical expression is given which just depending on this exciter system itself and not like mechanical resonant amplitude becomes higher to destroy the whole system at resonant 


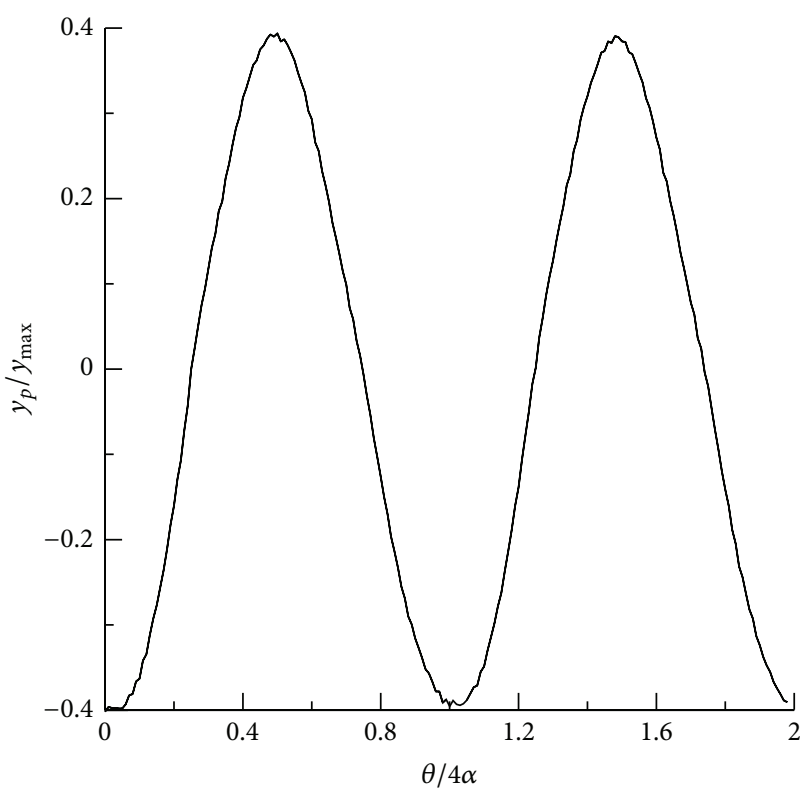

(a)

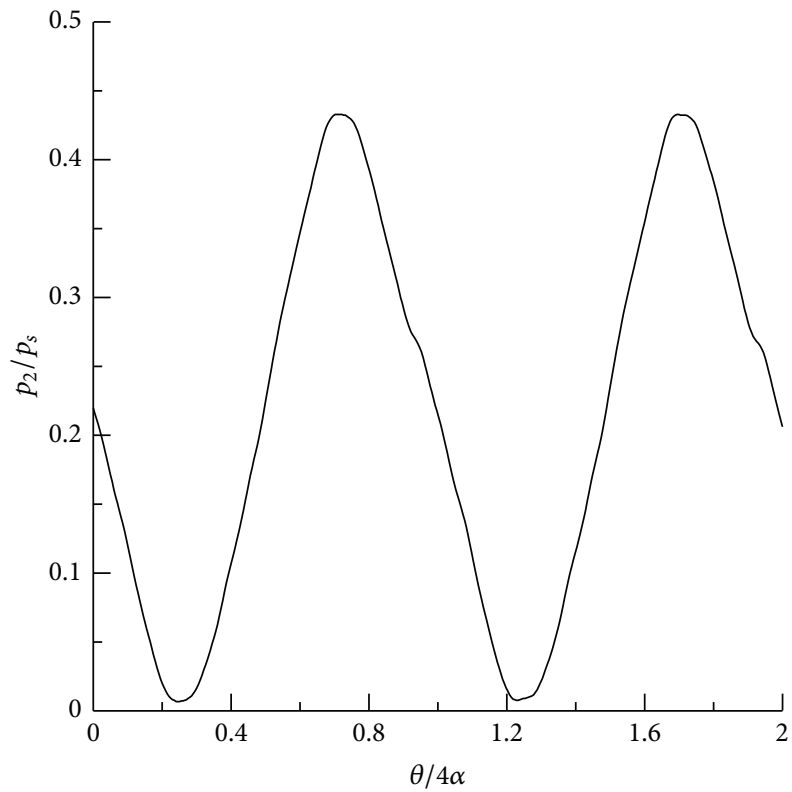

(c)

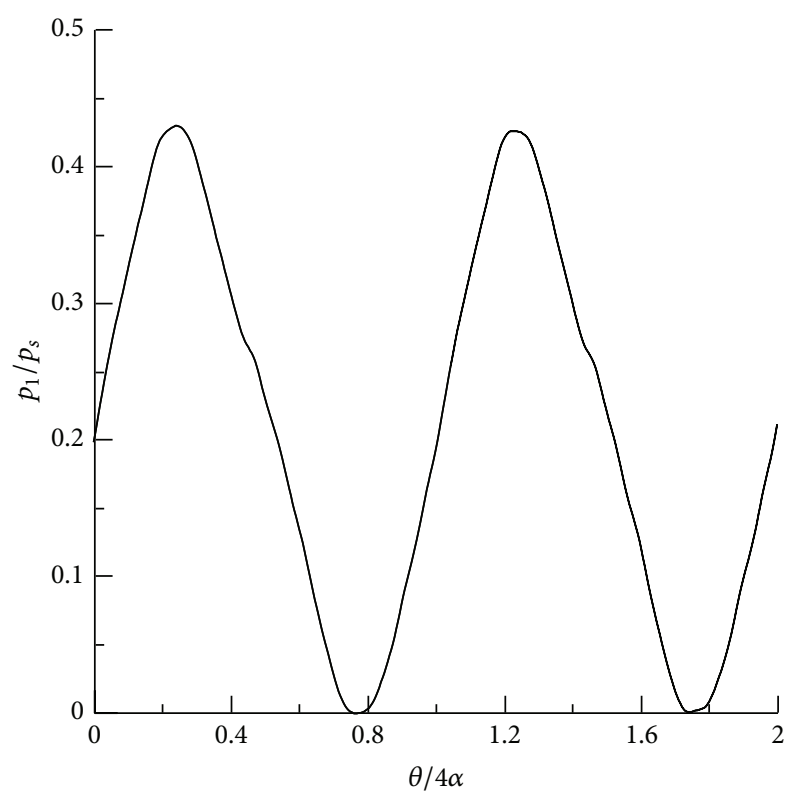

(b)

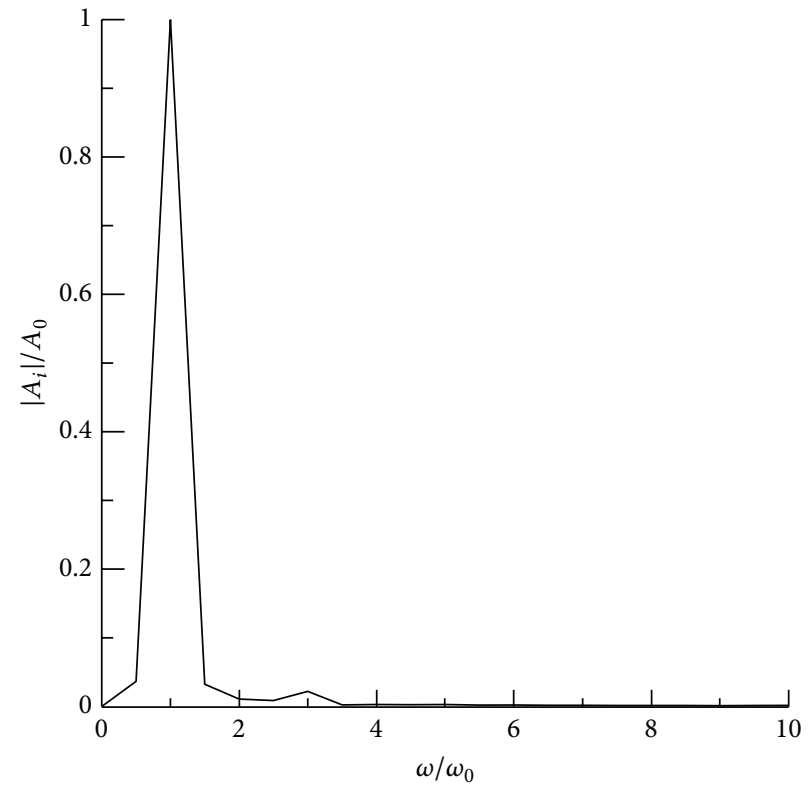

(d)

FIGURE 10: Resonant waveform $f=1800 \mathrm{~Hz}$ (a), pressure of the left chamber (b), pressure of the right chamber (c), and frequency composition (d).

frequency. The experimental system is built to verify the theoretical analysis. Though there is a coupling relationship between vibration amplitude and working frequency, this amplitude is suddenly enlarged to resonant peak at resonant frequency. Consequently, resonant energy can be used in high-cycle fatigue test to reduce the external input to this system and improve the vibration amplitude at high frequency.

\section{Conflict of Interests}

The authors declare that there is no conflict of interests regarding the publication of this paper.

\section{Acknowledgments}

The authors are grateful to the support from the Science Foundation of Department of Education of Zhejiang Province (no. Y201430397) and the Postdoctoral Science Foundation of China (no. 2013M541798).

\section{References}

[1] W. Schütz, "A history of fatigue," Engineering Fracture Mechanics, vol. 54, no. 2, pp. 263-300, 1996. 
[2] Y. Huang, S. Liu, and J. Zhao, "Optimal design of two-dimensional band-gap materials for uni-directional wave propagation," Structural and Multidisciplinary Optimization, vol. 48, no. 3, pp. 487-499, 2013.

[3] H. Tian, D. Fielden, M. J. Kirkham, and P. K. Liaw, "Control of noise and specimen temperature during $1 \mathrm{kHz}$ fatigue experiments," Journal of Testing and Evaluation, vol. 34, no. 2, pp. $92-$ 97, 2006.

[4] D. Lanning, G. K. Haritos, T. Nicholas, and D. C. Maxwell, "Low-cycle fatigue/high-cycle fatigue interactions in notched ti-6ai-4v," Fatigue and Fracture of Engineering Materials and Structures, vol. 24, no. 9, pp. 565-577, 2001.

[5] D. L. Davidson, J. B. Campbell, and R. A. Page, "The initiation and growth of fatigue cracks in a titanium aluminide alloy," Metallurgical Transactions A, vol. 22, no. 2, pp. 377-391, 1991.

[6] X. Du, D. Niu, and W. Liao, "Design and experimental studies on the inverse control magneto-rheological damper," Journal of Vibration and Shock, vol. 25, no. 5, pp. 49-53, 2006.

[7] J. M. Morgan and W. W. Milligan, "A $1 \mathrm{kHz}$ servohydraulic fatigue testing system," in Proceeding of the Conference High Cycle Fatigue of Structural Materials, pp. 305-312, Warrendale, $\mathrm{Pa}, \mathrm{USA}, 1997$.

[8] C.-T. Chen, J.-C. Renn, and Z.-Y. Yan, "Experimental identification of inertial and friction parameters for electro-hydraulic motion simulators," Mechatronics, vol. 21, no. 1, pp. 1-10, 2011.

[9] J. G. Hao and Y. C. Zhang, "Study on the properties of new electro-hydraulic exciting system," Journal of Taiyuan University of Technology, vol. 34, no. 6, pp. 706-709, 2003.

[10] Y. Ren and J. Ruan, "Regulating characteristics of an electrohydraulic vibrator multiply controlled by the combination of a two-dimensional valve and a standard servo valve," Proceedings of the Institution of Mechanical Engineers Part C: Journal of Mechanical Engineering Science, vol. 227, no. 12, pp. 2707-2723, 2013. 

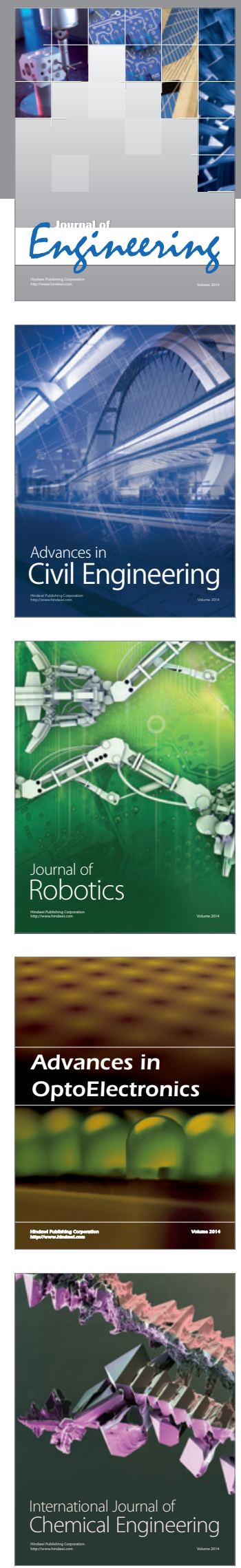

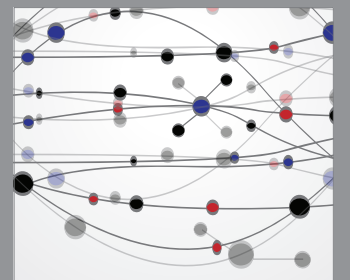

The Scientific World Journal
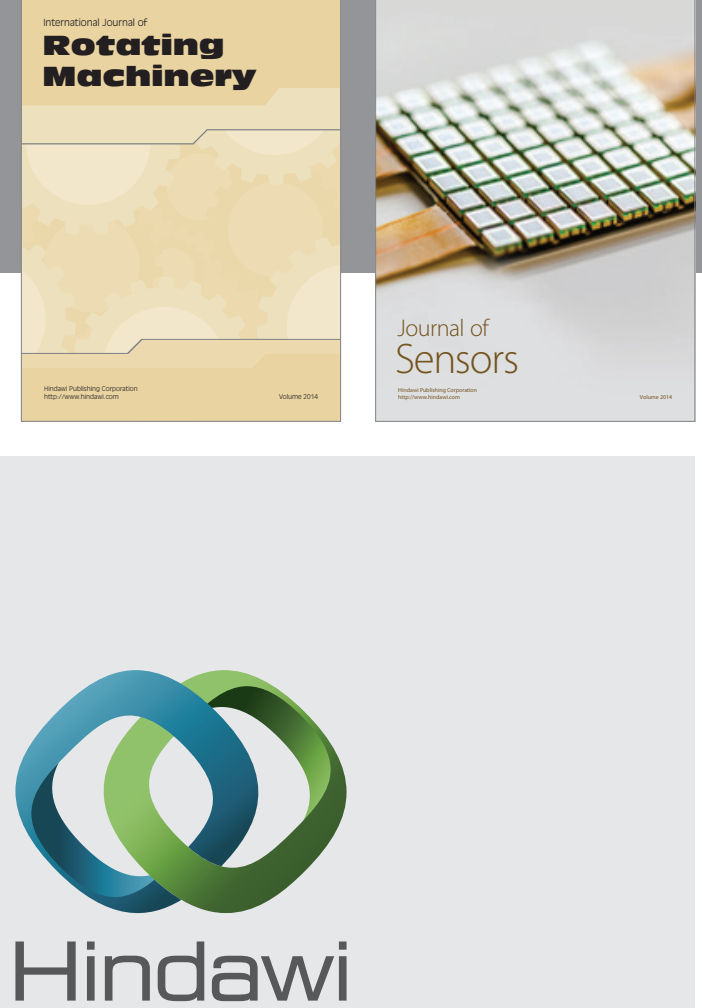

Submit your manuscripts at http://www.hindawi.com
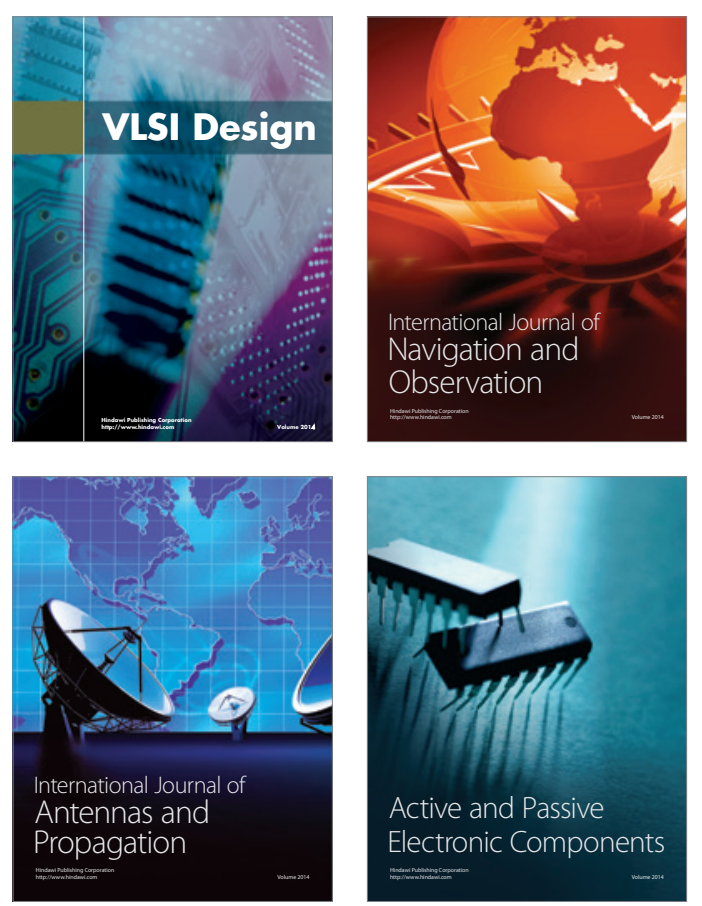
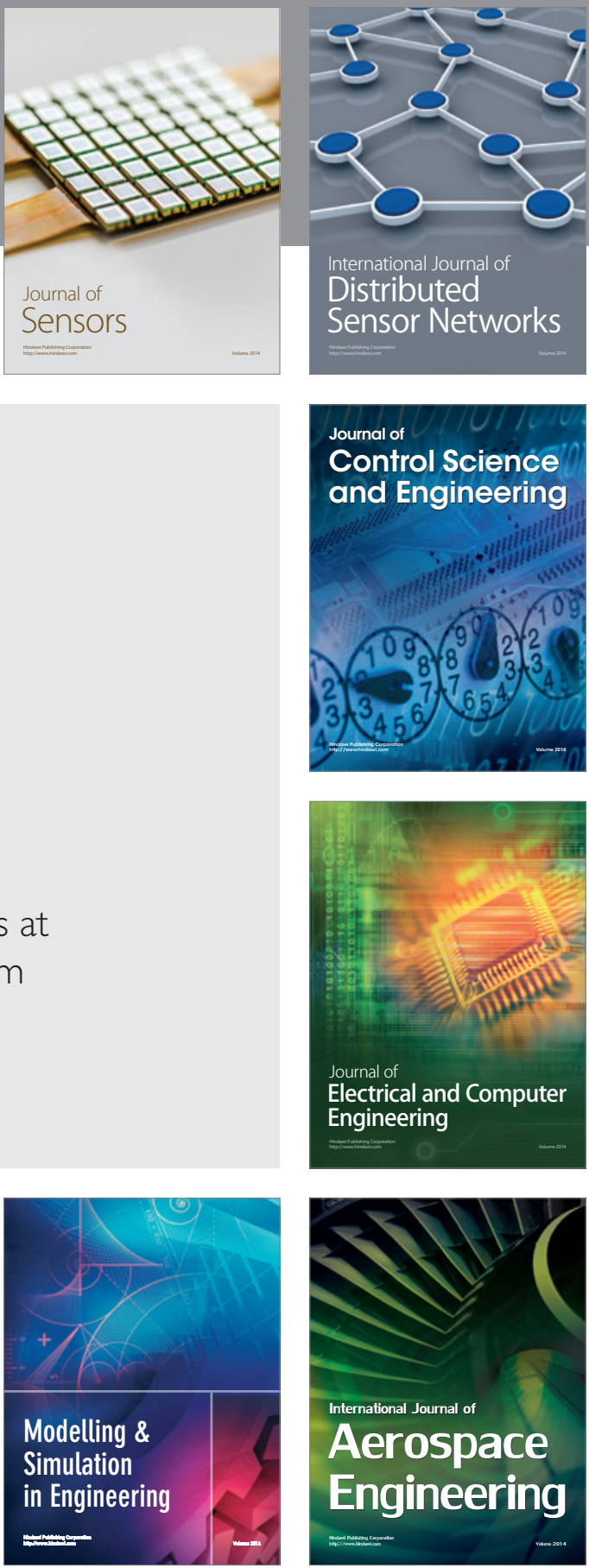

Journal of

Control Science

and Engineering
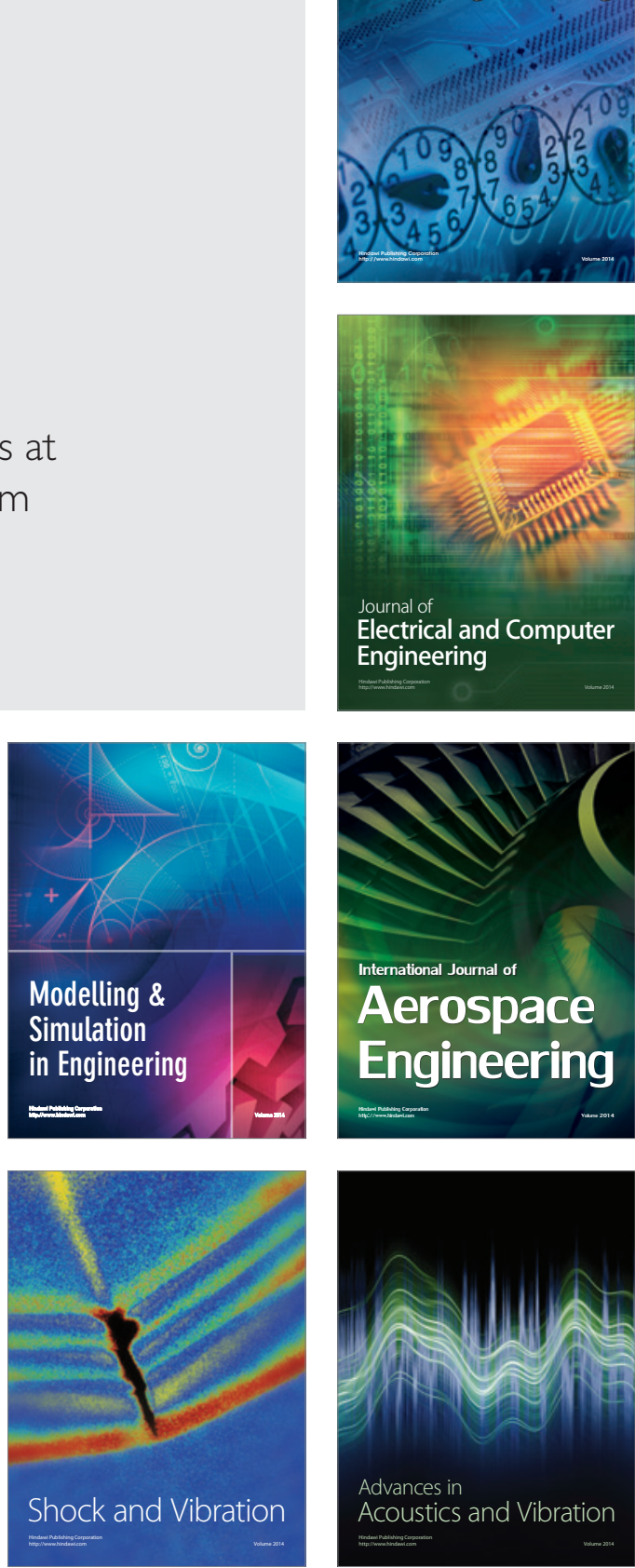